\title{
Image Saliency Detection Algorithm Based on Super Pixels Partition Wenwen Pan ${ }^{\mathrm{a}}$, Xiaofei Sun ${ }^{\mathrm{b}}$, Xia Wang ${ }^{\mathrm{c}}$, Tao Xu ${ }^{\mathrm{d}}$, Lina Gong ${ }^{\mathrm{e}}$ \\ College of Information Science and Engineering Zaozhuang University,Zaozhuang,277160,China \\ apanwenwen@vip.qq.com, b34740935@qq.com, 'c89141426@qq.com, d95185856@qq.com, ${ }^{\mathrm{e}} 390701387 @ q q . c o m$
}

Keywords: Saliency detection, super pixel, saliency map, salient object.

\begin{abstract}
This paper proposes an image saliency detection algorithm based on super pixels partition. The algorithm first uses the simple linear iterative clustering method to divide the image into multiple super pixels, and then according to the rules of global saliency to calculate the saliency level of each super pixel, finally the saliency map of the whole image is obtained. Experimental results show that the proposed approach can extract saliency map from the image of different size with different types of salient object, and the effect is better.
\end{abstract}

\section{Introduction}

The most important part of the image are often concentrated in the small key regions, these regions called salient region, image saliency detection task is to find out which regions of the image can attract the attention of human visual more ${ }^{[1]}$. Saliency map is used to reflect the different emphasis of human eye to different part of the image, the brighter regions can cause more attention of the human eye. With the loss of the brightness, the emphasis degree caused in turn reduce. Image saliency detection can be used for content transmission, target detection, image restoration, etc. The existing saliency detection methods are grouped into space-domain-based and frequency-domain- based ${ }^{[2]}$.Algorithms based on space domain are [3][4][5][6], the algorithms based on frequency domain are [7][8].

A saliency detection algorithm based on super pixels partition is proposed in this paper. Super pixel algorithm adopts the method of clustering groups pixels into meaningful atomic regions instead of pixel grid with fixed size and shape, in order to reduce computational complexity. And then according to the rules of global saliency to determine saliency degree of each super pixel. Finally generate saliency map with the saliency degree of each super pixel.

\section{Pixels Partition of Regionalization}

Existing super pixels partition algorithm can be grouped into two broad categories: graph-based algorithms and gradient-ascent-based algorithms. Super pixel partition algorithms based on graph theory have $[9,10,11]$ and based on the gradient descent algorithm have[12,13,14,9]. This article uses the simple linear iteration method of super pixel partition is described in the literature [15], namely the SLIC method.

SLIC is a super pixel partition algorithm based on $\mathrm{k}$ - means clustering algorithm. It has two important features: one is that through limiting the search space to an area which size is proportional to the the size of the super pixel to reduce the number of the distance calculation during the optimization process.The other is to weight the color and spatial proximity to control super pixel's size and close degree. Specific algorithm is as follows:

1) Initialize clustering center including Lab space, and $x, y$ pixel coordinates, and uniform the distribution of the clustering center according to the number of pixels fixed.

2) In order to prevent the clustering center from falling on the border the clustering center will be moved to the smallest gradient place within the $3 * 3$ neighborhood. 
3) Within $2 s * 2 s$ neighborhood of the cluster center distribue the matching point for each cluster center according to the set distance between each point and the clustering center to calculate.

4) Calculate the L1 distance between new clustering center and the clustering center before. According to the threshold value to judge whether need to disturb the clustering center, which is always iterative computation until the distance between the clustering center is less than a certain threshold.

5) End of operation

\section{Global Saliency Rules Determination}

The super pixels after image partition obtain regional characteristics. The super pixels instead of pixels to be calculated the degree of each part can greatly reduce the computing complexity. In order to determine the saliency degree of each super pixel compared with other super pixels in the whole image, we make two global saliency rules according to the characteristics of human visual attention as follows,

1) The region with larger contrast of color or brightness in the image can cause human's attention.

2) The influence of any other super pixels to one super pixel in the whole image increases with the color difference between them increasing, but decreases with the increase of distance difference.

According to global saliency rules, the degree of any super pixel spix influence on super pixel spix $_{j}$ is expressed with formula (1).

$$
\operatorname{dif}\left(\operatorname{spix}_{i}, \operatorname{spix}_{j}\right)=\frac{N \cdot d_{\text {color }}\left(\text { spix }_{i}, \text { spix }_{j}\right)}{c \cdot d_{\text {position }}\left(\operatorname{spix}_{i}, \text { spix }_{j}\right)}(i \neq j)
$$

$N$ is the number of super pixels after partition, $c$ is the coefficient to adjust the centroid distance.

The RGB color space is evenly distributed and Lab color space is evenly distributed, so the latter is more suitable for calculating the color distance of the image. In order to further reduce the computation complexity, quantify each image with 12 different grades in each color channel to thin the color space, and then calculate color distance between the two super pixels, as shown in formula (2).

$$
d_{\text {color }}\left(\text { spix }_{i}, \text { spix }_{j}\right)=\sum_{n_{1}=1}^{N_{1}} \sum_{n_{2}=1}^{N_{2}} p\left(c_{i, n_{1}}\right) p\left(c_{j, n_{2}}\right) d\left(c_{i, n_{1}}, c_{j, n_{2}}\right)
$$

Among them, $p\left(c_{i, n_{1}}\right)$ and $p\left(c_{j, n_{2}}\right)$ is respectively the probability of $n_{1}$ and $n_{2}$ color within super pixels spix $_{i}$, spix f $_{j}$ after the color sparse. $N_{1}, N_{2}$ is respectively the total number of color within super pixel spix $_{i}$, spix $_{j}$ after the color sparse.

Use barycentric coordinates Euclidean distance of two super pixels to indicate the difference of location between two super pixels, said as shown in formula (3).

$$
d_{\text {position }}\left(\text { spix }_{i}, \text { spix }_{j}\right)=\sqrt{\left(x_{\text {spix }_{i}}-x_{\text {spix }}\right)^{2}+\left(y_{\text {spix }}-y_{\text {spix }}\right)^{2}}
$$

Among them, $x_{\text {spix }}, y_{\text {spix }}$ and $x_{\text {spix }}, y_{\text {spix }}$ is respectively the $x, y$ centriod coordinate of super pixel spix $_{i}$, spix $_{j}$.

The final saliency value of super pixel spix $x_{i}$ is equal to the sum of the value that other super pixels $\operatorname{spix}_{j}(j \in\{1,2, \cdots, N\}$, 且 $j \neq i)$ influence on it, represented by formula (4).

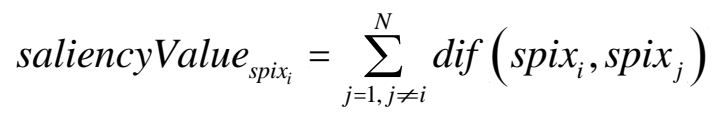




\section{Saliency Map Extraction and Comparative Analysis}

The method proposed in this paper is suitable for images with different size, and it can obtain good effect to extract saliency maps from images containing different types of salient objects. Not only can completely extract salient object, but also can well distinguish the saliency degree of each part of the salient object. In this paper, the salient objects are classified into four sorts: salient object with the color close to background's, small salient object, big salient object and multiple salient objects. Experiment on image library ImgSal ${ }^{[16]}$ and select one image of all kinds on their behalf. The original image and the corresponding saliency map is shown in Figure 1.

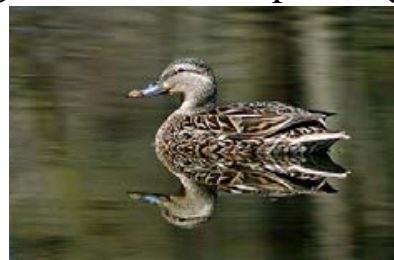

(a) salient object with the color close to background's
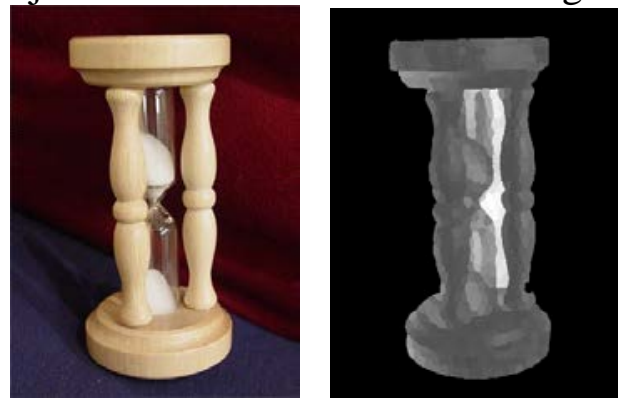

(c) big salient object
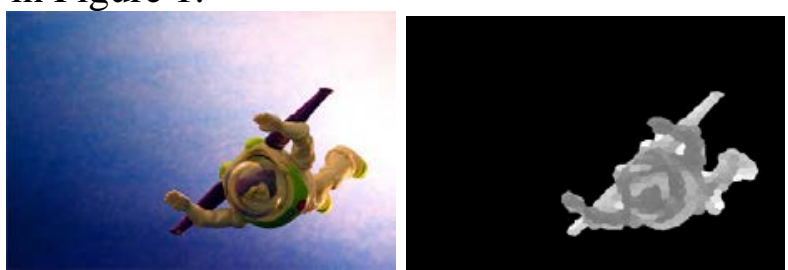

(b) small salient object
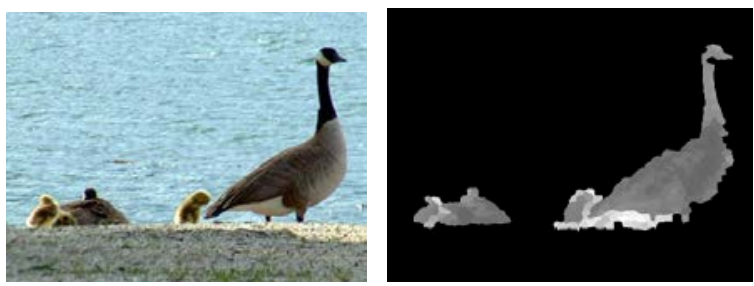

(d) multiple salient objects

Figure 1 Saliency map extracted on the basis of the SLIC super pixels partition

Compare the method proposed in this paper with the existing several saliency map extraction method, the result is shown in Figure 2. The saliency map extracted by IT algorithm ${ }^{[17]}$ is some discrete points. It cannot fully reflect the characteristics of the shape of the salient object. There is a widespread problem that hollow exists in the salient object within the saliency map extracted by MZ algorithm, GB algorithm and SR algorithm because only outline information of salient object is extracted. AC algorithmand IG algorithm can solve the problem of hollow effectively, but individual image background has higher saliency degree. The method proposed in this paper can better solve the problem of hollow, effectively suppress the influence of the background and well distinguish the saliency degree of each part of the salient object.

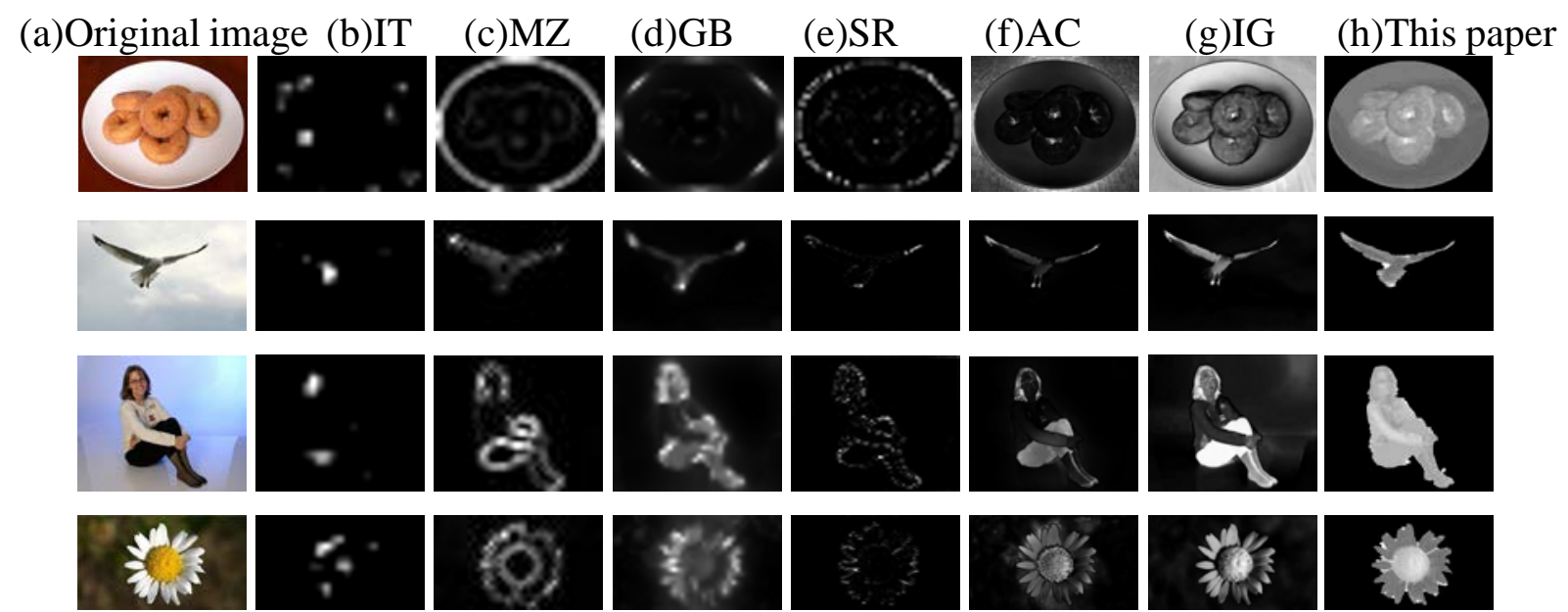

Figure 2 The comparison of saliency map extracted by different method 


\section{Conclusion and Prospect}

The saliency map extraction algorithm proposed in this paper uses the SLIC method to divide the image into different super pixels firstly, and then according to the rules of global saliency to determine saliency degree of each super pixel. It can extract saliency map from the image of different size with different types of salient object and achieve better effect than existing algorithm. Next, the method proposed in this paper will be considered to apply to image retrieval, image restoration, etc.

\section{Acknowledgments}

This work was financially supported by Zaozhuang university scientific research fund youth project. (Grant No.2015QN12)

\section{References}

[1] Wenwen Pan, Xiaofei Sun, Xia Wang, Wei Zhang. Brief Analysis on Typical Image Saliency Detection Methods [C]. International Conference on Information Sciences, Machinery, Materials and Energy 2015:1613-1618.

[2] Wenwen Pan, Xiaofei Sun, Xia Wang, Wei Zhang. Brief Analysis on Typical Image Saliency Detection Methods. International Conference on Information Sciences, Machinery, Materials and Energy (ICISMME), 2015.

[3] L. Itti, C. Koch, and E. Niebur. A model of saliency-based visual attention for rapid scene analysis. PAMI ,20(11):1254-1259,1998.

[4] Y.-F. Ma and H.-J. Zhang. Contrast-based image attention analysis by using fuzzy growing. ACM MM 2003.

[5] J. Harel, C. Koch, and P. Perona. Graph-based visual saliency. NIPS,19:545-552, 2007.

[6] R. Achanta, F. Estrada, P. Wils, and S. Süsstrunk. Salient region detection and segmentation. ICVS 2008.

[7] R. Achanta, S. Hemami, F. Estrada and S. Süsstrunk, Frequency-tuned Salient Region Detection, IEEE International Conference on Computer Vision and Pattern Recognition (CVPR 2009), pp. 1597 - 1604, 2009.

[8] X. Hou and L. Zhang. Saliency detection: A spectral residual approach. CVPR 2007.

[ 9 ] Yuhang Zhang, Richard Hartley, John Mashford and Stewart Burn, Superpixels via Pseudo-Boolean Optimization, International Conference on Computer Vision (ICCV), 2011.

[10] Ming-Yu Liu, Tuzel, O., Ramalingam, S. , Chellappa, R., Entropy Rate Superpixel Segmentation, CVPR, 2011.

[11] Pedro Felzenszwalb and Daniel Huttenlocher. Efficient graph-basedimage segmentation. International Journal of Computer Vision (IJCV),59(2):167-181, 2004.

[12] A. Levinshtein, A. Stere, K. Kutulakos, D. Fleet, S. Dickinson, and K. Siddiqi. Turbopixels: Fast superpixels using geometric flows. IEEETransactions on Pattern Analysis and Machine Intelligence (PAMI),2009.

[13] A. Vedaldi and S. Soatto. Quick shift and kernel methods for mode seeking. In European Conference on Computer Vision (ECCV), 2008.

[14] D. Comaniciu and P. Meer. Mean shift: a robust approach toward featurespace analysis. IEEE Transactions on Pattern Analysis and MachineIntelligence, 24(5):603-619, 2002.

[15] Radhakrishna Achanta, Appu Shaji, Kevin Smith Aurelien Lucchi, Pascal Fua, and Sabine Susstrunk[J]. SLIC Superpixels Compared to State-of-the-art Superpixel Methods. Joural of Latex Class Files, Vol. 6, NO. 1, 2011.

[16] AN X J, LRTINE L, HE H G, et al. ImgSal: A benchmark for saliency detection V1.0 [EB/OL]. (2001) [2013-12-21]. http://www.cim.mcgill.ca/ lijian/database.htm. 
[17] L. Itti, C. Koch, and E. Niebur. A model of saliency-based visual attention for rapid scene analysis. PAMI ,20(11):1254-1259,1998. 